\title{
Insuficiencia mitral moderada progresiva en un niño con síndrome de Axenfeld-Rieger. Importancia del seguimiento cardiológico
}

\author{
Progressive moderate mitral regurgitation in a children with Axenfeld-Rieger \\ syndrome. The importance of cardiologic follow up
}

Dr. Francisco Sánchez Ferrera y Lic. María D. Grima Murcia ${ }^{b}$

\section{RESUMEN}

El síndrome de Axenfeld-Rieger es una enfermedad congénita con una prevalencia estimada de 1 cada 200000 individuos. La afectación oftálmica con la disgenesia del segmento anterior es la que define la enfermedad. Se puede presentar desde el períodoneonatal. Seasocia con afectaciones extraoculares, como dismorfismo craneal y anomalías maxilofaciales o dentarias; otras manifestaciones menos frecuentes son las cardiológicas o hipofisarias. La aparición de cardiopatía no congénita en el síndrome de Axenfeld-Rieger ha sido descrita en muy pocos casos en la literatura.

Presentamos un paciente de 7 años de edad que presentó síndrome de Axenfeld-Rieger, con insuficiencia mitral ligera desde los 3 años que progresó a insuficiencia mitral moderada en la actualidad.

El seguimiento cardiológico estaría indicado en pacientes con síndrome de Axenfeld-Rieger.

Palabras clave: sindrome de Axenfeld-Rieger, insuficiencia de la válvula mitral, cardiopatías, grupo de salud interdisciplinario.

\begin{abstract}
Axenfeld-Rieger syndrome is a congenital disease with an estimated prevalence of one in 200,000 individuals. This is an ophthalmic disorder related to anterior segment dysgenesis, which may be present from the neonatal period. It is associated with extraocular affectations such as cranial dimorphism, maxillofacial or dental anomalies. Cardiological or pituitary manifestations are less common. The congenital heart disease in Axenfeld-Rieger syndrome has been described in very few cases in the literature.
\end{abstract}

a. Unidad de Cardiología Pediátrica. Servicio de Pediatría. Hospital Universitario de San Juan de Alicante, Alicante, España.

b. Instituto de Bioingeniería y Centro de Investigación Biomédica en Red en Bioingeniería, Biomateriales y Nanomedicina (CIBER-BBN). Universidad Miguel

Hernández, Alicante, España.

Correspondencia:

Dr. Francisco Sánchez Ferrer: pacosanchezferrer0@hotmail.com

Financiamiento: Ninguno.

Conflicto de intereses: Ninguno que declarar.

Recibido: 12-2-2016

Aceptado: 11-5-2016
We reporta 7-year-old patient with Axenfeld-Rieger syndrome and mild mitral insufficiency since the age of 3 years, which is progressing to moderate mitral regurgitation at the present time. The cardiologic follow up may be indicated in patients with Axenfeld-Rieger syndrome.

Key words: Axenfeld-Rieger syndrome, mitral regurgitation, heart diseases, interdisciplinary health team.

http:/ /dx.doi.org/10.5546/aap.2016.e417

\section{INTRODUCCIÓN}

El síndrome de Axenfeld-Rieger (SAR) es una enfermedad rara congénita descrita por el oftalmólogo Theodor Axenfeld en 1920, que se define por alteraciones de la cámara anterior ocular asociadas a otras afecciones sistémicas. ${ }^{1-3}$ Se puede clasificar como anomalía de Axenfeld (limitada a defectos oftálmicos del segmento anterior), anomalía de Rieger (en la que se producen cambios adicionales en el iris) o síndrome de Rieger (anomalías oculares y defectos de desarrollo extraoculares, en especial, de los dientes, los huesos faciales y la piel periumbilical). Debido al solapamiento genotípico y fenotípico, estas enfermedades son consideradas bajo el nombre de SAR. Estas tres variantes son ahora reconocidas como un espectro del mismo síndrome.

La prevalencia estimada es de 1 cada 20000 personas, con herencia autosómica dominante, con alta penetrancia, pero con variabilidad intrafamiliar. Se muestran mutaciones en los genes PITX2 y FOXC1, que son los responsables de la enfermedad, ${ }^{4,5}$ identificados en el $40 \%-60 \%$ de los casos. ${ }^{6}$

Exponemos el caso de un niño de 7 años con SAR, con diagnóstico neonatal por afectación oftálmica. En su evolución presentó a los tres años una insuficiencia mitral ligera, que no tenía en el primer año de vida, con progresión desde el diagnóstico a insuficiencia mitral moderada. 
Si bien el SAR es muy poco prevalente y la afectación cardíaca congénita no es frecuente, la afectación cardíaca no congénita progresiva, como es el caso presentado, es excepcional.

\section{CASO CLÍNICO}

Presentamos a un niño de 7 años con SAR en seguimiento desde el nacimiento, cuando fue diagnosticado por afectación ocular en el período neonatal. Antecedentes de madre y hermana mayor del paciente con SAR. A nivel oftalmológico, el paciente presentaba miopía magna, estrabismo, coloboma bilateral y varios episodios de glaucoma en ambos ojos de ángulo abierto. Precisó tratamiento médico con gotas oftálmicas, un beta-bloqueante no selectivo y un análogo sintético de las prostaglandinas. En el ojo derecho, ante el fracaso del tratamiento médico, necesitó cirugía ocular en 5 ocasiones hasta el momento, mediante trabéculo-trabeculectomía.

En el período neonatal, presentó un soplo cardíaco de intensidad I/VI y, dada la asociación de cardiopatía con el SAR, se realizó una ecocardiografía, donde se mostró un foramen oval permeable (FOP) y una estenosis pulmonar ligera con una válvula de aspecto normal. Los controles posteriores mostraron un cierre del FOP y una normalización en los gradientes de salida de la válvula pulmonar. A los 3 años, en una revisión, se observó una insuficiencia mitral ligera que previamente no presentaba y, a los 6 años de edad, el paciente se encontraba asintomático sin tratamiento y la ecocardiografía mostraba una progresión de la insuficiencia mitral a moderada, con un flujo de regurgitación central con una vena contracta de $3 \mathrm{~mm}$ que llegaba hasta la cúpula de la aurícula izquierda; el flujo de insuficiencia ocupaba aproximadamente un tercio de la cavidad auricular. No se observaron otras alteraciones ecocardiográficas; el aspecto de los músculos papilares y valvas mitrales era normal, sin prolapso. Actualmente, a los 7 años, ya observamos dilatación de la aurícula izquierda.

Se realizan controles en consulta con Cardiología Pediátrica cada 6 meses, junto con el hospital de referencia para cirugía cardíaca infantil. Dado que se encuentra asintomático, la actitud es expectante, y se indica únicamente la profilaxis de la endocarditis.

Por la característica evolutiva que ha mostrado la insuficiencia mitral, es esperable que continúe progresando y que se produzca una mayor dilatación de la aurícula izquierda y sintomatología clínica. En ese caso, precisaría tratamiento quirúrgico de la válvula, que podría ser reparada mediante cirugía o reemplazada. El momento indicado para la cirugía debe ser el más cercano posible al fin del desarrollo físico del paciente, lo que optimiza, hasta este momento, el tratamiento médico en caso de precisarlo.

Con 7 años, el niño se encuentra escolarizado en el año correspondiente a su edad, sin dificultades académicas. El desarrollo psicomotor ha sido normal hasta el momento, así como la integración social y familiar del paciente. Por ello, no ha presentado ninguna otra afectación del SAR, salvo la afectación ocular y cardíaca descrita.

En el estudio de extensión familiar, se

FIGURA 1. Imagen ecográfica de la insuficiencia mitral moderada y dilatación auricular izquierda
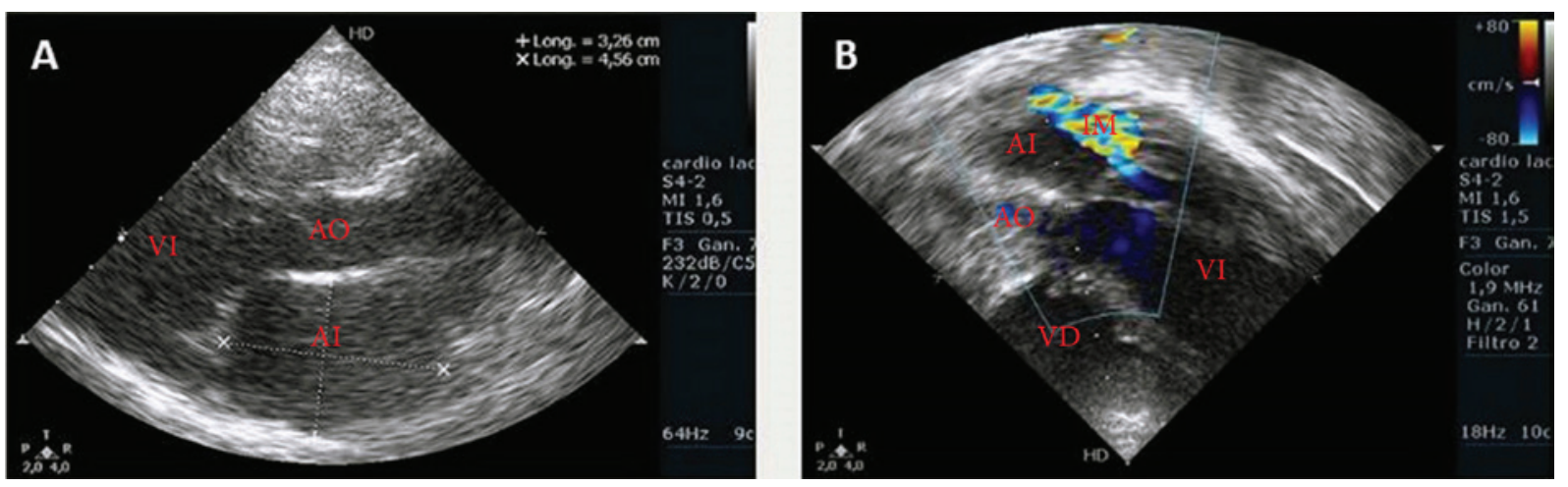

A: ecocardiografía paraesternal, eje largo del paciente; se observa la aurícula izquierda dilatada (+3,1 puntaje Z) con una relación aurícula izquierda-aorta de 1,9. B: ecocardiografía de 5 cámaras con doppler color que muestra regurgitación de la válvula mitral. Ao: aorta; AI: aurícula izquierda; VI: ventrículo izquierdo; VD: ventrículo derecho; IM: insuficiencia mitral. 
realizó un estudio cardiológico (no realizado previamente) a la hermana de 16 años con diagnóstico de comunicación interauricular amplia tipo ostium secundum de $16 \mathrm{~mm}$, con dilatación de cavidades derechas, que precisó cierre percutáneo. El estudio materno fue normal.

La genética realizada mediante el kit de sondas Salsa P054 (incluyen FOXL, FOX1, FOX2, PITX2, OA1) no mostró alteraciones, como en la mitad de los pacientes con SAR. El consejo genético a la familia, dado que es una enfermedad autosómica dominante con alta penetrancia, consiste en explicar al paciente del caso y a su hermana que los hijos que tengan presentan, aproximadamente, un $50 \%$ de probabilidad de manifestar SAR, si bien las afectaciones extraoculares que presenten son difíciles de predecir, debido a la variabilidad clínica del síndrome.

\section{DISCUSIÓN}

El SAR, clínicamente, se manifiesta por anomalías oculares desde el nacimiento, que afectan, sobre todo, al iris con hipoplasia de este, corectopia o policoria. La córnea puede ser afectada con un desplazamiento prominente y anterior de la línea de Schwalbe (embriotoxón posterior). ${ }^{7}$ Esta dismorfogénesis ocasiona incrementos de la presión ocular, lo que da lugar a glaucoma $^{8}$ en el $50 \%$ de los pacientes. ${ }^{9}$

Además de la afectación ocular, se ha asociado dismorfismo craneal, anomalías maxilofaciales con hipoplasia mandibular, afectación dentaria con microdoncia e hipodoncia y redundancia de piel periumbilical. ${ }^{2,5,9}$ Más raras son otras afectaciones, como las cardíacas, en las que se han definido, clásicamente, defectos del septo interauricular, así como alteraciones valvulares en tractos de salida ventricular. ${ }^{10-13}$ También han sido descritas afectaciones hipofisarias con retraso del crecimiento ${ }^{13}$ o hipospadia. ${ }^{3}$

El seguimiento continuado en Oftalmología es fundamental para evitar la presión intraocular elevada y el glaucoma, si bien no hay que descuidar las afectaciones sistémicas descritas.

Nuestro paciente con SAR presentaba alteraciones oftalmológicas de coloboma bilateral, estrabismo, miopía magna y glaucoma de ángulo abierto, y precisó tratamiento médico y múltiples cirugías. El diagnóstico fue precoz, ya que es una patología autosómica dominante y los antecedentes familiares de la madre y la hermana mayor con el síndrome sugirieron su investigación diagnóstica desde el nacimiento. ${ }^{4}$ Se estudió la genética asociada a este síndrome, que fue negativa, como ocurre en, aproximadamente, la mitad de los casos. ${ }^{6}$

Además de las afecciones habituales, en nuestro caso, queremos destacar la importancia del seguimiento cardiológico. Las malformaciones más frecuentes, como alteraciones del septo interauricular (como presentaba su hermana) o alteraciones en el tracto de salida ventriculares, se pueden evaluar desde el período neonatal, pues son malformaciones congénitas. ${ }^{12,14}$ En cambio, en nuestro paciente, fue a partir de los 3 años cuando se manifestó una insuficiencia mitral que fue progresiva con el paso de los años.

La insuficiencia mitral no congénita progresiva no es frecuente en la edad pediátrica, por lo que consideramos que puede estar asociada al SAR.

En la literatura, se ha presentado, recientemente, un caso de insuficiencia mitral con falla cardíaca en un niño de 21 meses con este síndrome y alteraciones en el gen FOXC1 ${ }^{10}$ y en otro paciente de 33 años con insuficiencia mitral grave..$^{15}$ En la literatura actual, el SAR ha sido relacionado con cardiopatías septales o valvulares congénitas asociadas, principalmente, a los genes descritos, como el FOXC1, si bien estas asociaciones no nos ayudan a entender la fisiopatología de la progresión de la insuficiencia mitral en nuestro caso.

Resaltamos la necesidad, en todos los casos, del estudio cardiológico inicial a todo paciente con SAR para descartar las alteraciones cardíacas, como el defecto del septo interauricular o alteraciones valvulares ya descritas.

El presente caso se suma a la literatura que sugiere una correlación entre alteraciones cardiológicas (en este caso, insuficiencia mitral) y el SAR. Por lo tanto, creemos conveniente que los pacientes con SAR se sometan a exámenes ecocardiográficos seriados.

\section{REFERENCIAS}

1. Shields MB. Axenfeld-Rieger syndrome: a theory of mechanism and distinctions from the iridocorneal endothelial syndrome. Trans Am Ophthalmol Soc 1983;81:736-84.

2. Dressler S, Meyer-Marcotty P, Weisschuh N, JablonskiMomeni A, et al. Dental and craniofacial anomalies associated with Axenfeld-Rieger Syndrome with PITX2 mutation. Case Rep Med 2010;2010:621984.

3. Jorgenson RJ, Levin LS, Cross HE, Yoder F, et al. The Rieger syndrome. Am J Med Genet 1978;2(3):307-18.

4. Tümer Z, Bach-Holm D. Axenfeld-Rieger syndrome and spectrum of PITX2 and FOXC1 mutations. Eur J Hum Genet 2009;17(12):1527-39.

5. Maciolek NL, Alward WLM, Murray JC, Semina EV, et al. Analysis of RNA splicing defects in PITX2 mutants supports a gene dosage model of Axenfeld-Rieger syndrome. BMC Med Genet 2006;7:59. 
6. Hjalt TA, Semina EV. Current molecular understanding of Axenfeld-Rieger syndrome. Expert Rev Mol Med 2005;7(25):1-17.

7. Ito YA, Goping IS, Berry F, Walter MA. Dysfunction of the stress-responsive FOXC1 transcription factor contributes to the earlier-onset glaucoma observed in Axenfeld-Rieger syndrome patients. Cell Death Dis 2014;5:e1069.

8. Idrees F, Vaideanu D, Fraser SG, Sowden JC, et al. A review of anterior segment dysgeneses. Surv Ophthalmol 2006;51(3):213-31.

9. Shields MB. Axenfeld-Rieger syndrome: a theory of mechanism and distinctions from the iridocorneal endothelial syndrome. Trans Am Ophthalmol Soc $1983 ; 81: 736-84$

10. Gripp KW, Hopkins E, Jenny K, Thacker D, et al. Cardiac anomalies in Axenfeld-Rieger syndrome due to a novel FOXC1 mutation. Am J Med Genet A 2013;161A(1):114-9.
11. Bekir NA, Güngör K. Atrial septal defect with interatrial aneurysm and Axenfeld-Rieger syndrome. Acta Ophthalmol Scand 2000;78(1):101-3.

12. Swiderski RE, Reiter RS, Nishimura DY, Alward WL, et al. Expression of the Mf1 gene in developing mouse hearts: implication in the development of human congenital heart defects. Dev Dyn 1999;216(1):16-27.

13. Meyer-Marcotty P, Weisschuh N, Dressler P, Hartmann $\mathrm{J}$, et al. Morphology of the sella turcica in AxenfeldRieger syndrome with PITX2 mutation. J Oral Pathol Med 2008;37(8):504-10.

14. Akkus MN, Argin A.Congenital heartdefects in two siblings in an Axenfeld-Rieger syndrome family. Clin Dysmorphol 2010;19(2):56-61.

15. AntevilJ,UmakanthanR,LeaccheM,BrewerZ,etal.Idiopathic mitral valve disease in a patient presenting with AxenfeldRieger syndrome. J Heart Valve Dis 2009;18(3):349-51. 